\title{
Synthesis and Size-dependent Optical Properties of Intermediate Band Gap $\mathrm{Cu}_{3} \mathrm{VS}_{4}$ Nanocrystals
}

\author{
Valeria Mantella, ${ }^{\dagger}$ Silviya Ninova, ${ }^{\S}$ Seryio Saris, ${ }^{\dagger}$ Anna Loiudice, ${ }^{\dagger}$ Ulrich Aschauer, ${ }^{\S}$ and Raffaella \\ Buonsanti $^{\text {+*}^{*}}$ \\ ${ }^{\dagger}$ Laboratory of Nanochemistry for Energy (LNCE), Department of Chemical Sciences and Engineering, École Poly- \\ technique Fédérale de Lausanne, $\mathrm{CH}-1950$ Sion, Switzerland \\ ${ }^{\S}$ Department of Chemistry and Biochemistry, University of Bern, Bern, Switzerland
}

\begin{abstract}
Intermediate band gap semiconductors are an underexplored class of materials with unique optical properties of interest for photovoltaic and optoelectronic applications. Herein, we synthesize highly crystalline cubic $\mathrm{Cu}_{3} \mathrm{VS}_{4}$ nanocrystals with tunable edge length of $9 \mathrm{~nm}, 12 \mathrm{~nm}$ and $18 \mathrm{~nm}$. Since size-control is achieved for the first time for this semiconductor, particular emphasis is dedicated to the structural/compositional analysis, the formation mechanism and the size-dependent optical properties. The corresponding UV-Vis spectra reveal three absorption peaks in the visible range, resulting from the intermediate band gap electronic structure of $\mathrm{Cu}_{3} \mathrm{VS}_{4}$, which blue-shift with decreasing size. Density functional theory reveals these size-dependent optoelectronic properties to result mostly from weak quantum confinement. The reported results pave the way towards further fundamental investigations of the physicochemical properties of intermediate band gap semiconductors in the nanoscale regime for solar energy harvesting.
\end{abstract}

\section{INTRODUCTION}

Colloidal semiconductor nanocrystals (NCs) have been studied for almost 30 years and their size and shape dependent properties combined with their rich surface chemistry continue to intrigue a large scientific community. Both fundamental studies and practical applications (i.e. light emitting diodes and third generation photovoltaics) are profiting from the tunability achieved through colloidal chemistry, which spans from precise size control of binary NCs (i.e. CdSe, PbSe) to geometry (i.e. core@shell, heterodimers) and composition control in multinary NCs. ${ }^{1-}$ ${ }^{3}$ The latter include ternary metal chalcogenides and perovskite NCs, which exemplify the increasing importance of composition as a tool to manipulate the optoelectronic properties in addition to size. ${ }^{1-9}$ Ternary metal chalcogenides possess a very rich phase diagram with highly tunable electronic properties that allows for materials emitting in the visible and near-IR without the use of toxic metals. ${ }^{6-8}$ Within this class of materials, remarkable attention has been devoted to $\mathrm{Cu}$-based ternary chalcogenides: $\mathrm{CuInS}_{2}$ (CIS) NCs are the most investigated system to date by virtue of the fact that both size and stoichiometry $(\mathrm{Cu} / \mathrm{In}$ ratio) can be modulated to improve the photoluminescence quantum yield and to tune the band gap over a wide energy range. ${ }^{9-11}$ Cesium lead bromide NCs and their derivatives have recently attracted a great deal of interest for their compelling properties related to their ionic bonding and they have reached state-of-the-art photovoltaics performance in just a few years. ${ }^{12-14}$
One additional class of ternary compounds with peculiar optoelectronic properties are the intermediate band gap (IB) semiconductors. ${ }^{15-17}$ These materials possess allowed mid-gap states within their fundamental band gap that enable a more efficient absorption of solar irradiation. ${ }^{15^{-17}}$ Indeed, in addition to the electronic transitions initiated by the absorption of higher energy photons, whose energy is comparable to the fundamental band gap, sub-bandgap energy photons can also be absorbed to promote electrons from the valence band (VB) to the IB and from the IB to the conduction band (CB). The IB semiconductors offer an interesting approach to up-conversion, which could potentially increase the conversion efficiency imposed by the Shockley-Queisser limit up to $63.1 \%$ under full concentration, yet they have not been largely explored so far..$^{15-18}$ The formation of mid-gap states has been achieved via several methods such as the use of quantum nanostructures, highly mismatched alloys and high concentrations of impurities. ${ }^{19}$ The characteristic IB electronic structure has also been observed in semiconductors containing transition metal atoms, such as $\mathrm{Fe}^{20}, \mathrm{~V}^{21}$ and $\mathrm{Cr}^{22}$ For instance, Ghosh et al. showed that in $\mathrm{CuFeS}_{2}$ the intermediate states in the fundamental gap arise from empty Fe $3 \mathrm{~d}$ states by using density functional theory (DFT).$^{20}$ Chen et al. reported similar results for $\mathrm{Cr}$-doped $\mathrm{CuGaS}_{2}$, where the density of states (DOS) analysis revealed that the IB is dominated by $\mathrm{Cr} 3 \mathrm{~d}$ states. ${ }^{22}$ DFT-based methods have predicted $\mathrm{Cu}_{3} \mathrm{VS}_{4}$, also known as sulvanite, to possess an IB electronic structure which, jointly with its composition made solely of earth-abundant elements, make $\mathrm{Cu}_{3} \mathrm{VS}_{4}$ an interesting candidate for solar energy harvesting. ${ }^{23,24,25,26}$ 
Among the IB semiconductors, only $\mathrm{CuFeS}_{2}$ has been synthesized in the form of colloidal NCs, and these studies have already evidenced intriguing physico-chemical properties and new opportunities for this novel class of $\mathrm{NCs}^{20,27,28}$ For instance, $\mathrm{CuFeS}_{2} \mathrm{NC}^{2}$ were employed as efficient photothermal agents in the biological window of 6509oonm due to their small size and strong near infrared (NIR) absorption. ${ }^{20}$ It was also demonstrated that for $\mathrm{CuFeS}_{2}$, the optical transition between the VB and the IB is contributing to the permittivity allowing for quasi-static plasmonic resonances in the UV-vis range, contrarily to the expectation that dielectric materials should not exhibit plasmon resonances. ${ }^{27}$ Furthermore, one of the parameters which currently limits the sub-band gap photocurrent in quantum dots IB solar cells fabricated by epitaxial growth techniques is the spatial density of the dots, something which could be easily overcome using a colloidal ink. ${ }^{16}$

Herein, we report a colloidal hot-injection method to synthesize highly uniform, cube-shaped colloidal $\mathrm{Cu}_{3} \mathrm{VS}_{4} \mathrm{NCs}$ with three different sizes $(9 \mathrm{~nm}, 12 \mathrm{~nm}$ and $18 \mathrm{~nm}$ ). Since size-control is achieved for the first time for this semiconductor, particular emphasis is dedicated to the structural/compositional analysis and to the formation mechanism. The phase purity of the $\mathrm{Cu}_{3} \mathrm{VS}_{4} \mathrm{NCs}$ is assessed by means of X-Ray Diffraction (XRD), Raman spectroscopy and High-Resolution Transmission Electron Microscopy (HRTEM). The stoichiometry of the $\mathrm{Cu}_{3} \mathrm{VS}_{4} \mathrm{NCs}$ is found to be size dependent with $\mathrm{Cu}$-rich surfaces leading to an increasing $\mathrm{Cu}$ content as the NCs size decreases. Investigation of the reaction mechanism by XRD and TEM reveals an in-situ seed mediated growth with $\mathrm{Cu}_{\mathrm{x}} \mathrm{S}$ (where $\mathrm{x}=1,2$ ) particles reacting with the vanadium molecular precursor. Three distinct absorption bands are observed in the UVVis spectra and are ascribed to electronic transitions occurring from the valence band (VB) to the intermediate band (IB). DFT calculations evidence that the synthesized NCs fall in a weak quantum confinement regime which mostly explains the peak shifts observed in the spectra. The results herein open new perspectives for the synthesis and understanding of the optical properties of IB nanomaterials and, more generally, of novel copper-based semiconductors, including $\mathrm{CsCu}_{5} \mathrm{X}_{3}(\mathrm{X}=\mathrm{S}, \mathrm{Se}, \mathrm{Te}){ }^{29}$

\section{EXPERIMENTAL SECTION}

\section{General}

All glassware was oven-dried prior to use. Standard Schlenk line techniques assisted by a nitrogen filled glovebox were used for all the synthesis. A J-KEM Scientific Model 310 temperature controller was used with a heating mantle for reaction temperature control.

Materials. Copper (I) iodide (CuI, 98\%), vanadyl acetylacetonate (VO(acac) $\left.)_{2}, 98 \%\right)$, oleylamine (OLAM, technical grade, $70 \%$ ), trioctylamine (TOA, 98\%), Oleic acid (OLAC, technical grade, 90\%), 1-octadecene (ODE, technical grade, $90 \%$ ), trioctylphosphine (TOP, technical grade 90\%), 1-dodecanethiol (DDT, technical grade 98\%), ethanol (anhydrous) were all purchased from Sigma-Aldrich and used as received without further purification. Hexane (anhydrous, 95\%) was purchased from TCI Deutschland $\mathrm{GmbH}$.

Synthesis of $18 \mathrm{~nm}, 12 \mathrm{~nm}$ and $9 \mathrm{~nm}$. $\mathrm{Cu}_{3} \mathrm{VS}_{4} \mathrm{NCs} \mathrm{Cu}_{3} \mathrm{VS}_{4}$ $\mathrm{NCs}$ were synthetized by utilizing the $\mathrm{CuFeS}_{2}$ synthesis reported by Ghosh et al. as a starting point ${ }^{20}$ In a typical synthesis, CuI (1mmol, o.19og), TOP (1mmol, $0.446 \mathrm{~mL}$ ) and $\mathrm{VO}(\mathrm{acac})_{2}$ ( $\left.1.33 \mathrm{mmol}, \mathrm{o} .35 \mathrm{og}\right)$, were dissolved in ODE $(7 \mathrm{ml})$, were introduced in a $25 \mathrm{ml}$ three-necked round-bottom flask under a protective atmosphere of nitrogen which was maintained during the whole synthesis. The resulting mixture was stirred and heated up at $14^{\circ} \mathrm{C} / \mathrm{min}$ to $280^{\circ} \mathrm{C}$ for the $18 \mathrm{~nm}, 260^{\circ} \mathrm{C}$ for the $12 \mathrm{~nm}$ and $250^{\circ} \mathrm{C}$ for the $9 \mathrm{~nm}$ NCs. In the meanwhile, DDT (10mmol, 2.395ml) was kept under nitrogen at room temperature for 20 minutes. Degassed OLAM (3mmol, $1 \mathrm{ml})$ was then added to DDT and the resulting solution was swiftly injected into the metal precursor solution at the target temperature. It is very important to avoid any condensation inside the reaction environment and to maintain a homogenous mixing. Indeed, the different local temperature gradients would lead to a broader dispersion of NCs sizes. After injection, the temperature was allowed to recover and the final solution was held at the targeted temperature for 30 minutes. After this time, the reaction vessel was let to spontaneously cool down to room temperature by removing the heating mantle. The resulting nanocrystals were washed using a mixture of ethanol and hexane (1:3) to remove unreacted precursors and/or excess of ligands. After three cycles of centrifugation, the precipitate was dispersed in anhydrous hexane and stored in glovebox. The use of the anhydrous solvent allows to preserve the colloidal stability for months. Instead, the NCs stored in hydrous hexane precipitate within one day. No compositional change was observed when the solution is stored in air.

\section{X-Ray Diffraction (XRD) Analysis.}

The XRD patterns reported were acquired on a Bruker D8 Advance diffractometer with a $\mathrm{Cu} \mathrm{K} \alpha$ source equipped with a Lynxeye one-dimensional detector. The diffractometer operated at $40 \mathrm{kV}$ and $40 \mathrm{~mA}$ with a $\mathrm{Cu} \mathrm{K} \alpha$ source with wavelength of $\lambda=1.54 \AA$. The samples were drop-casted on a silicon wafer, previously washed using acetone and isopropanol.

\section{Electron Microscopy.}

Transmission Electron Microscopy (TEM) images were recorded on an Analytical JEOL-210oF FETEM using a beam energy of $120 \mathrm{kV}$, equipped with a Gatan camera. Samples were drop-casted on a copper TEM grid (Ted Pella, Inc.) prior to imaging. Size statistics were performed using the software ImageJ and counting 200 particles per sample. Elemental mapping was performed on 10 particles per samples and the error on the atomic \% estimated to be $0.1 \%$. High-resolution TEM (HR-TEM) images, STEMHAADF (scanning transmission electron microscopy high angle annular dark-field) images and corresponding EDX (energy-dispersive X-ray spectroscopy) maps were acquired on a FEI Tecnai-Osiris using an accelerating voltage 
of $200 \mathrm{kV}$. This microscope is equipped with a high brightness X-FEG gun, silicon drift Super-X EDX detectors and a Bruker Esprit acquisition software.

\section{Raman spectroscopy.}

Raman spectra were recorded using a confocal Raman microscope (Renishaw inVia Raman microscope) with a 532 $\mathrm{nm}$ laser source of maximum power of $45 \mathrm{~W}$. The measurements were performed with a 1oox objective in the Raman microscope with $5 \%$ of laser power. The spectral positions were calibrated by the characteristic Si phonon peak at $520.7 \mathrm{~cm}^{-1}$.

\section{Steady Sate UV-Vis-NIR Absorption Spectroscopy.}

Optical extinction spectra of dilute hexane dispersions of the NC samples were performed in transmission mode using a Perkin Elmer- Lambda 1050 UV/Vis/NIR Spectrophotometer with a $\mathrm{D}_{2}$ (deuterium) lamp for the ultraviolet range and a WI (halogen) lamp for the visible and near infrared range.

\section{Scanning Electron Microscopy (SEM).}

SEM images were acquired on a FEI Teneo 200 FEG Analytical Scanning Electron Microscope using a beam energy of $5 \mathrm{keV}$.

\section{X-Ray Photoelectron Spectroscopy (XPS)}

XPS was performed using VersaProbe II from Physical Electronics. Analysis was carried out using a monochromatic $\mathrm{Al} \mathrm{K \alpha} \mathrm{X}$-ray source operating at $25 \mathrm{~W}$ with a beam size of $100 \mu \mathrm{m}$. The spherical mirror analyzer was set at $45^{\circ}$ take-off angle with respect to the sample surface. The binding energy of the spectra were calibrated by setting the $\mathrm{C}$ $\mathrm{C}$ bound of the $\mathrm{C}$ is peak at $284.8 \mathrm{eV}$.

\section{DFT Calculations.}

The DFT calculations were carried out using the Vienna $a b$ initio simulation package, VASP ${ }^{30,31}$ using the $\mathrm{PBEsol}^{32}$ ex- $^{-}$ change-correlation functional. Geometry relaxation was performed with a force threshold of $0.01 \mathrm{eV} / \AA$. We used a plane-wave cut-off of $400 \mathrm{~V}$ and projector-augmented wave potentials with $\mathrm{Cu}$ (4s, 3d), V (4s, 3d) and S (3s, 3P) valence states to describe the core-valence electron interactions. As confirmed in the experimental section, $\mathrm{Cu}_{3} \mathrm{VS}_{4}$ crystallizes in the cubic $\mathrm{P}-43 \mathrm{~m}$ space group. We performed volume relaxation and found a lattice parameter of $5.312 \AA$, which is in reasonable agreement with experiment (5.39 $\AA$ ). ${ }^{33,34}$ In comparison, a previous study by Kehoe et al. found a value of $5.358 \AA \AA^{23,24} \mathrm{~A} \Gamma$-centered k-mesh with dimension $8 \times 8 \times 8$ was used to sample the Brillouin zone. A Hubbard U parameter of $5.2 \mathrm{eV}$ was applied on the $\mathrm{Cu} \mathrm{d}$ states in agreement with other theoretical calculations ${ }^{23,24}$ and similar to other $\mathrm{Cu}^{+}$compounds, such as $\mathrm{Cu}_{2} \mathrm{O}{ }^{35} \mathrm{In}$ order to study the optical properties of the compounds, we calculated the frequency-dependent dielectric function within the independent particle approximation. This consists of an imaginary part $\left(\varepsilon_{2}\right)$, determined as a summation over conduction band states, and a real part $\left(\varepsilon_{1}\right)$, obtained from the Kramers-Kronig transformation. For structural relaxations, we used an electronic smearing of $0.01 \mathrm{eV}$, which for the calculations of the spectra was increased to $0.2 \mathrm{eV}$. The absorption coefficient was then determined as:

$$
\alpha=\frac{4 \pi}{\lambda} \sqrt{\left[\frac{\sqrt{\left(\varepsilon_{1}^{2}+\varepsilon_{2}^{2}\right)}-\varepsilon_{1}}{2}\right]}
$$

\section{RESULTS AND DISCUSSION}

\section{Structural and compositional characterization}

$\mathrm{Cu}_{3} \mathrm{VS}_{4} \mathrm{NCs}$ were synthesized by means of colloidal synthesis. Copper (I) iodide (CuI), vanadyl acetylacetonate $\left(\mathrm{VO}(\mathrm{acac})_{2}\right)$ and 1-dodecanethiol (DDT) were chosen as precursors for $\mathrm{Cu}$, $\mathrm{V}$ and S, respectively. Trioctylphosphine (TOP) and oleylamine (OLAM) were selected as the ligands to control size and shape, respectively. Our approach relies on the injection of DDT and OLAM into a hot solution containing $\mathrm{Cu}$ and V precursors along with TOP and 1-octadecene (ODE) as the solvent. Figure 1 shows TEM images of typical samples obtained at different injection temperatures. The NCs exhibit a cubic shape and possess a size ranging from $9 \mathrm{~nm}$ to $18 \mathrm{~nm}$ (standard deviations less than $15 \%$ ) depending on the reaction temperature $\left(250^{\circ} \mathrm{C}, 260^{\circ} \mathrm{C}\right.$ and $280^{\circ} \mathrm{C}$ for $9 \mathrm{~nm}, 12 \mathrm{~nm}$ and $18 \mathrm{~nm}$, respectively).
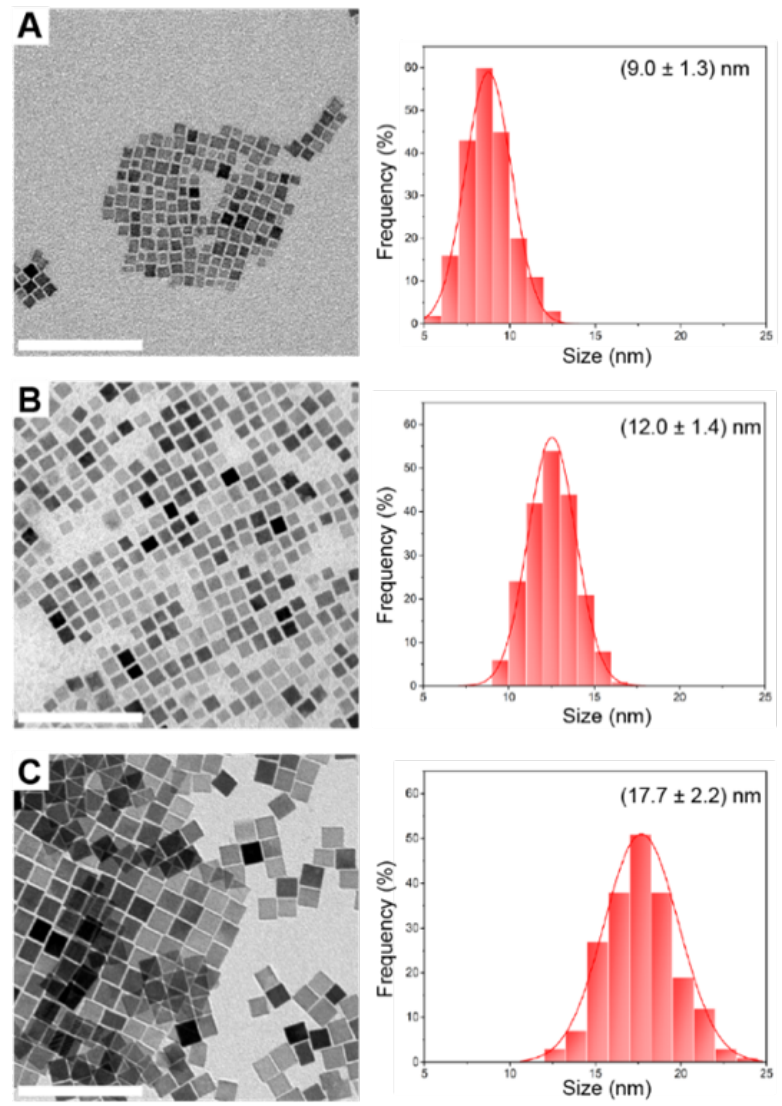

Figure 1. TEM images and corresponding statistical size analyses of $\mathrm{Cu}_{3} \mathrm{VS}_{4} \mathrm{NCs}$ synthesized at different temperatures: A) at $250^{\circ} \mathrm{C}$ separated from an insoluble precipitate (which constitutes a small fraction of the sample), B) at $260^{\circ} \mathrm{C}$ and C) at $280^{\circ} \mathrm{C}$. The scale bar is 1oonm. 
The crystalline phase was assessed by X-Ray diffraction (Figure $2 \mathrm{~A})$ which reveals the pure cubic $\mathrm{Cu}_{3} \mathrm{VS}_{4}(P-43 m, a=$ 5.393(1) $\AA$ ) phase for the three samples with a peak broadening consistent with the size of the crystallites being in the nanoscale regime. No other crystalline phases, including other existing stoichiometries of copper vanadium sulfide (Figure S1), copper sulfide, vanadium sulfide and copper, were detected. As a further confirmation to the phase purity, the $\mathrm{Cu}_{3} \mathrm{VS}_{4} \mathrm{NCs}$ were analyzed by Raman spectroscopy where a good agreement between the experimental and reference peaks is observed (Figure $2 \mathrm{~B}$ ).

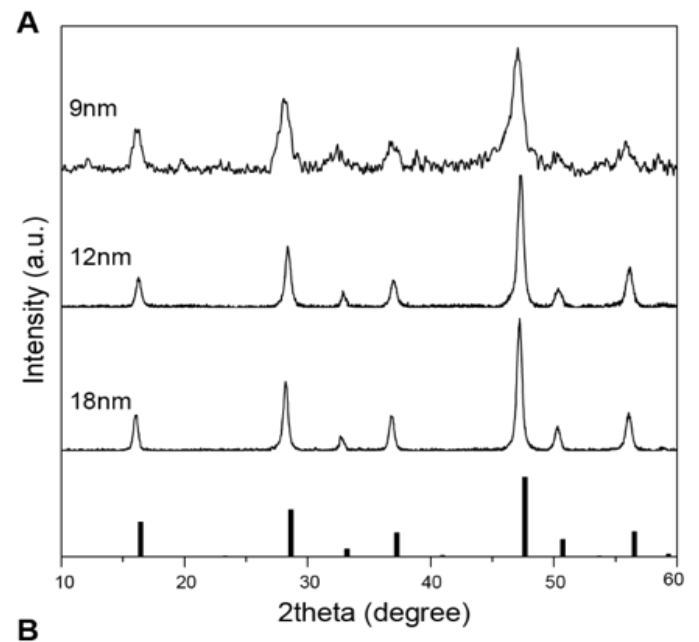

B

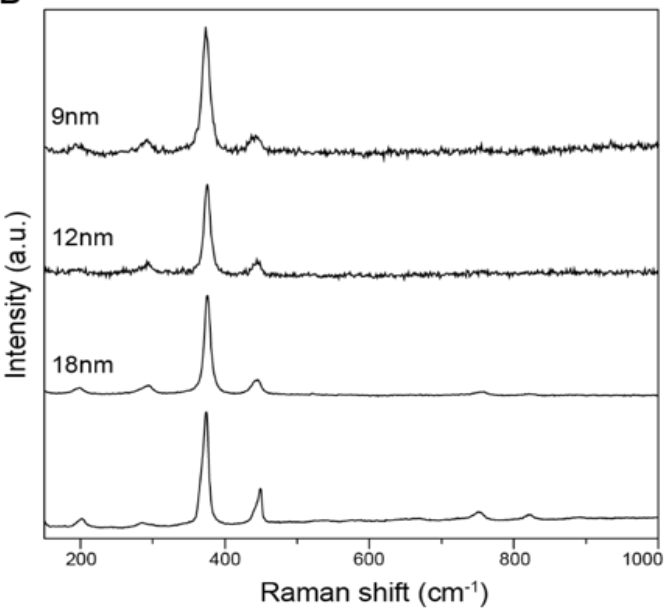

Figure 2. A) XRD patterns and B) Raman spectra of the assynthetized $9 \mathrm{~nm}, 12 \mathrm{~nm}, 18 \mathrm{~nm} \mathrm{Cu}_{3} \mathrm{VS}_{4} \mathrm{NCs}$. The $\mathrm{Cu}_{3} \mathrm{VS}_{4}$ XRD reference pattern (PDF-01-o88-1318) as well as the Raman reference spectrum (Ro60853) are reported at the bottom of each panel.

In conjunction with the XRD and Raman data, HRTEM along with Fast Fourier Transform (FFT), (Figures 3A, B), evidence the single crystalline nature and the cubic structure of the $\mathrm{Cu}_{3} \mathrm{VS}_{4} \mathrm{NCs}$. To investigate the composition of the $\mathrm{Cu}_{3} \mathrm{VS}_{4}$ NCs, scanning transmission electron microscopy-energy dispersive X-ray spectrometry (STEM-EDX), (Figure 3D-F), was deemed to be the most reliable technique. In fact, ICP results were not consistent, probably because of difficulties with sample cleaning or because of the well-known problems with sulfur digestion (formation of volatile $\mathrm{H}_{2} \mathrm{~S}$ ). Elemental mapping analysis performed on at least 10 particles per sample revealed an increase in the copper content as the NC size decreases. Specifically, the $\mathrm{Cu}: \mathrm{V}: \mathrm{S}$ ratio was $5: 1: 4,4.6: 1: 4$ and $4: 1$ : 4 for the $9 \mathrm{~nm}, 12 \mathrm{~nm}$ and $18 \mathrm{~nm}$ NCs, respectively, with standard deviations on the elemental ratios within the uncertainty of the EDX analysis which is $3-4 \%$.
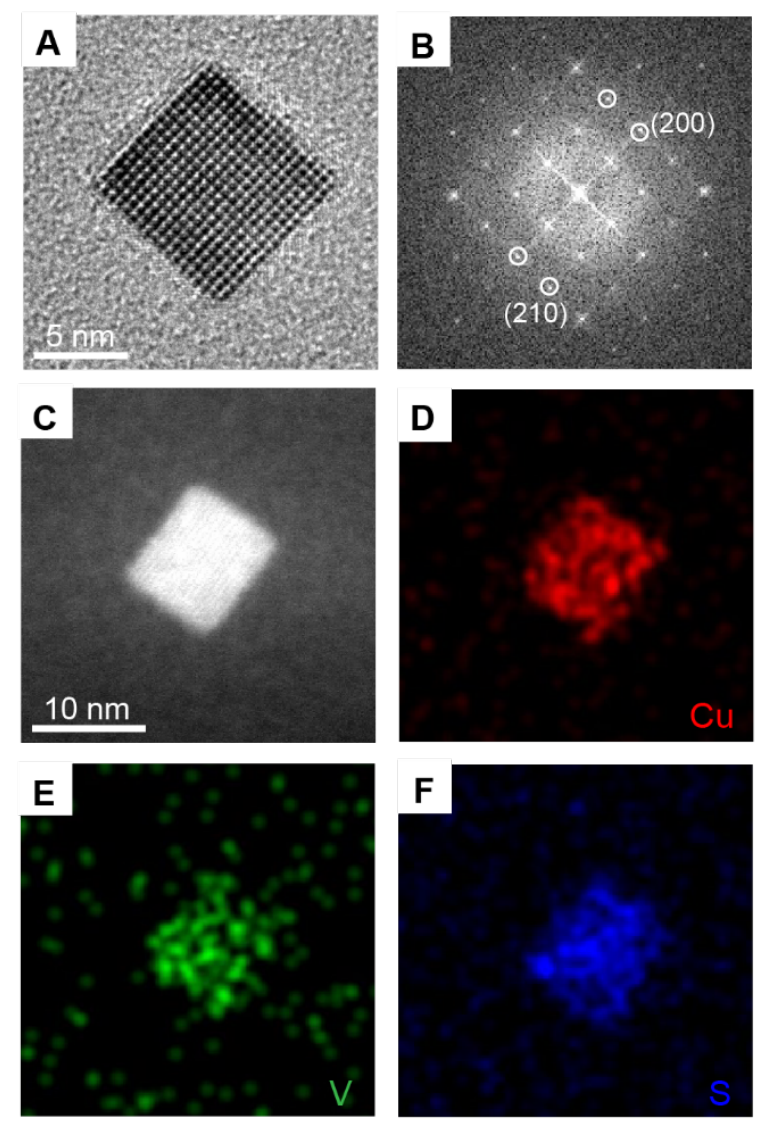

Figure 3. A, B) High- Resolution TEM images along with the FFTs of the $12 \mathrm{~nm}$ sample. C-F) HAADF-STEM image and elemental mapping of the same sample.

As mentioned in the case of CIS, off-stoichiometries are common in ternary semiconductors. For the $\mathrm{Cu}_{3} \mathrm{VS}_{4} \mathrm{NCs}$, three options were considered to explain the excess copper content: i) different crystalline phase (i.e. $\mathrm{Cu}_{5} \mathrm{VS}_{4}$ ), ii) interstitial $\mathrm{Cu}$ ions and iii) surface enrichment ("Cu-shell model"). To assess the first hypothesis, XPS was performed (Figure S2). The analysis indicates the presence of $\mathrm{S}^{-2}$ and of $\mathrm{Cu}^{+1}$ but it is not straightforward for the vanadium (see Supporting Information for detailed discussion). Because no crystalline phase associated with a $\mathrm{Cu}$-rich stoichiometry has been reported so far and the XRD pattern perfectly matches the $\mathrm{Cu}_{3} \mathrm{VS}_{4}$, the hypothesis of a different phase is excluded at the moment. In the cubic $\mathrm{Cu}_{3} \mathrm{VS}_{4}$ unit cell, the apices as well as the middle of the edges are vacant and create a $3 \mathrm{D}$ channel system along the [100] directions. Arribart et al proposed interstitial $\mathrm{Cu}^{+}$ions, with their very high mobility of $10^{-4} \mathrm{~cm}^{2} \mathrm{~V}^{-1} \mathrm{sec}^{-1}$ at $300 \mathrm{~K}$, to be the most probable candidates to intercalate into these tetrahedral vacant sites. ${ }^{23,36}$ However, this hypothesis would not explain the size-dependent compositional changes. Alternatively, a $\mathrm{Cu}$-rich surface would correlate well to an increase of the $\mathrm{Cu}$ content as the 


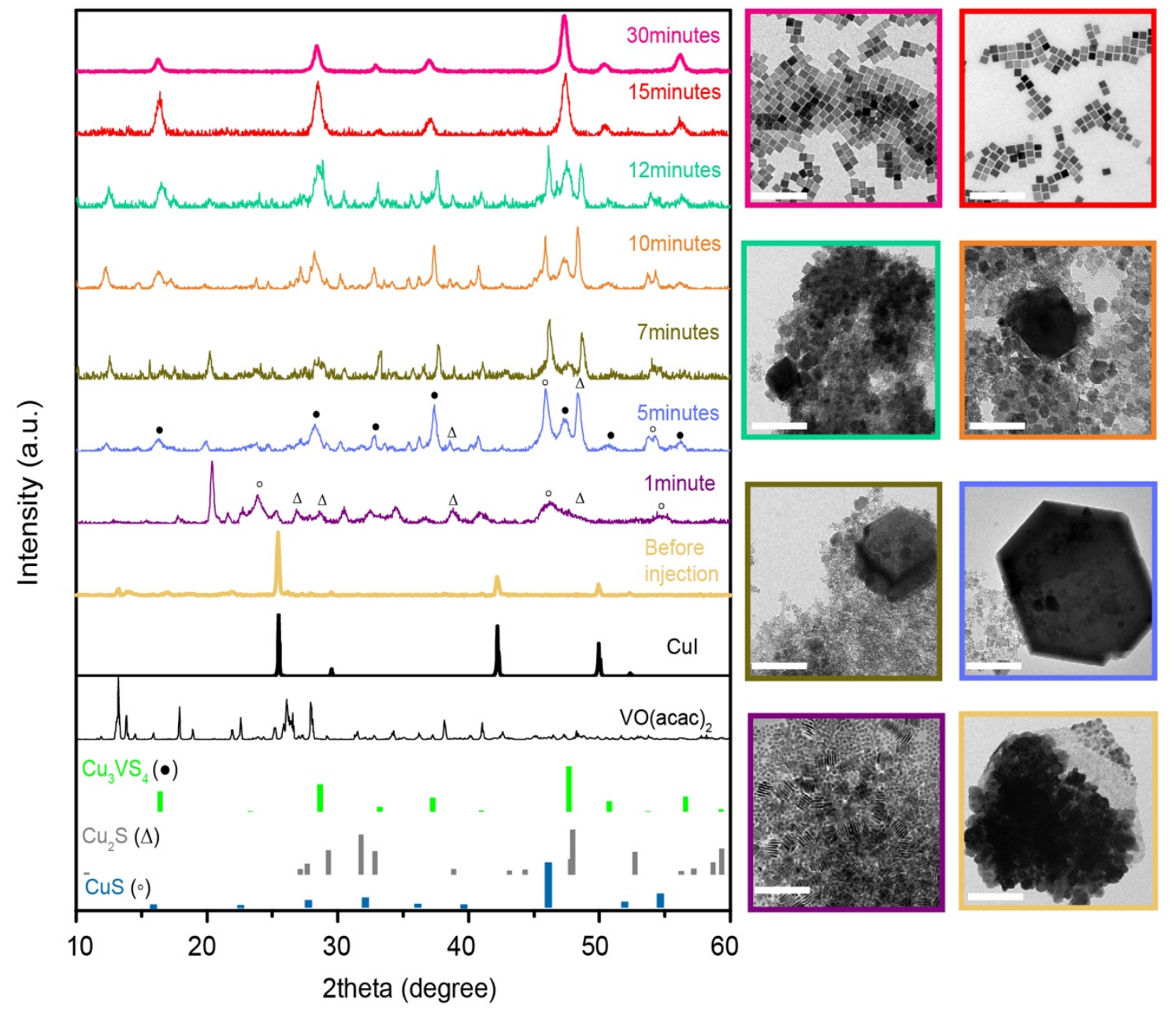

Figure 4. XRD patterns and TEM images of aliquots extracted from the reaction flask at different times during the synthesis of $\mathrm{Cu}_{3} \mathrm{VS}_{4}$ performed at $280^{\circ} \mathrm{C}$. Reference patterns of the $\mathrm{CuI}$ and $\mathrm{VO}(\mathrm{acac})_{2}$ powders, $\mathrm{Cu}_{3} \mathrm{VS}_{4}(\mathrm{PDF}$ o1-o88-1318), Cu $\mathrm{S}$ (PDF oo-0530522) and CuS (PDF 04-004-6505) are shown at the bottom of the XRD panel. The scale bars in the TEM images are 200 nm.

size decreases (i.e. surface-to-volume ratio increases). To assess this hypothesis, the non-stoichiometric model developed by Wang et al for PbSe NCs was applied to the $\mathrm{Cu}_{3} \mathrm{VS}_{4} \mathrm{NCs}$ (see details in the Supporting Information). ${ }^{37} \mathrm{~A} \mathrm{Cu}$ shell of $0.42 \mathrm{~nm}$, which corresponds to three monolayers of $\mathrm{Cu}$ atoms (134pm), was calculated for all the NC sizes. To find the same value, regardless of the size, confirms that the non-stoichiometry derives from a Cu-rich surface. Different thicknesses would not necessarily follow the observed size-dependent trend.

\section{Study of the $\mathrm{Cu}_{3} \mathrm{VS}_{4} \mathrm{NCs}$ growth mechanism}

When developing novel synthetic approaches, understanding the role played by the different reaction parameters is crucially important to advance the general knowledge in materials chemistry. Insights into the synthesis of multinary compounds are particularly needed, considering the challenges related to the incorporation of three or more different chemical elements in a homogeneous composition. In kineticallydriven synthetic techniques, like colloidal chemistry, slight variations in the chemical potentials of the species involved in the nucleation and growth can lead to the crystallization of undesired phases. In particular, the precursor reactivity must be carefully tuned in order to avoid the formation of amorphous phases (too low reactivity) or phase segregation (too high reactivity). Based on hard and soft (Lewis) acids and bases (HSAB) theory, the stronger bond between $\mathrm{Cu}^{+}$(soft Lewis acid) and $\mathrm{I}^{-}$(soft Lewis base) is expected to slow down the reaction of $\mathrm{Cu}^{+}$with the $\mathrm{S}$ source (R-SH, soft Lewis base) and ultimately prevent aggregation and precipitation of the resulting nanoparticles, as it was previously demonstrated for the CuInS ${ }_{2}$ synthesis. ${ }^{11}$ For what concerns the transformation process of $\mathrm{VO}(\text { acac })_{2}$, the formation of an intermediate $\mathrm{V}-\mathrm{S}$ complex is excluded for two main reasons: the first one is that $\mathrm{V}^{4+}$ is a hard Lewis acid, hence, it forms an unstable complex with $\mathrm{S}$ and the second reason is that the two bulky bidentate ligands (acetylacetonates) hinder the nucleophilic attack of the thiol to the metallic cationic core. Experimentally, no crystalline product was isolated when reacting $\mathrm{VO}$ (acac $)_{2}$ in absence of the $\mathrm{Cu}$ precursor under the same conditions of the $\mathrm{Cu}_{3} \mathrm{VS}_{4}$ 


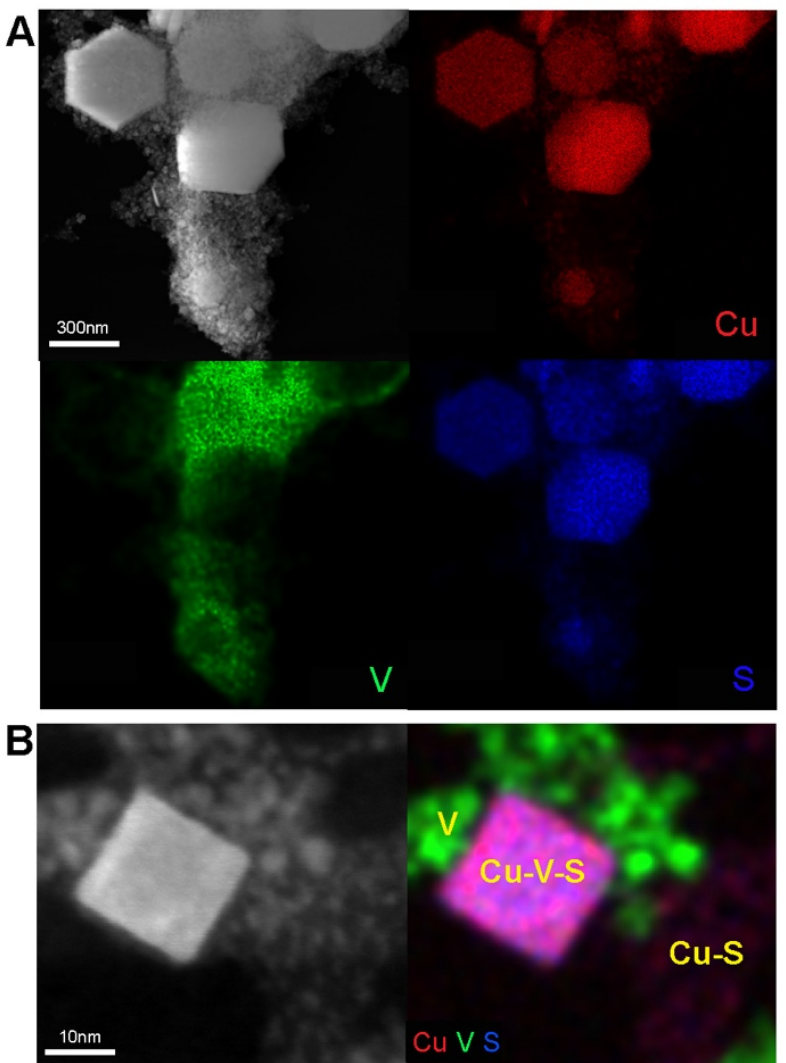

Figure 5. A, B) HAADF-STEM images and elemental mapping of the reaction product obtained after 5 minutes from the injection.

synthesis. However, small particles were detected in the TEM (FigureS3), which most likely are amorphous vanadium containing species from the $\mathrm{VO}(\mathrm{acac})_{2}$ decomposition. To gain valuable insights into the reaction mechanism, several aliquots were extracted, during the synthesis at scheduled time intervals, and quenched in ODE. The corresponding XRD patterns and TEM images are reported in Figure 4. A progressive and distinctive phase change is observed. At time zero, the intense signal from the undissolved $\mathrm{CuI}$ precursor dominates the XRD pattern. Interestingly, 1 minute after the injection of DDT and OLAM in the reaction mixture, XRD highlights the presence of covellite $\mathrm{CuS}$ and chalcocite $\mathrm{Cu}_{2} \mathrm{~S}$ together with other small peaks which can be assigned to the $\mathrm{VO}(\text { acac })_{2}$ or its decomposition products. ${ }^{38-40}$ Two different morphologies (nanospheres and nanoplatelets) of $\mathrm{Cu}_{\mathrm{x}} \mathrm{S}$ nanoparticles are observed in TEM and, based on a size selective precipitation, the nanospheres can be assigned to chalcocite $\mathrm{Cu}_{2} \mathrm{~S}$ and the nanoplatelets to covellite $\mathrm{CuS}$. After 5 minutes the cubic $\mathrm{Cu}_{3} \mathrm{VS}_{4}$ phase appears in the XRD pattern and concomitantly a few nanocubes are observed in the corresponding TEM image. As the reaction proceeds, the intensity of the XRD peaks of $\mathrm{Cu}_{3} \mathrm{VS}_{4}$ increases relatively to the $\mathrm{Cu}_{2} \mathrm{~S}$ and $\mathrm{CuS}$ and the population of nanocubes in the TEM images increases. Simultaneously, the shrinkage of copper sulfide nanoparticles is visible in the corresponding TEM images. These results point towards the $\mathrm{Cu}_{\mathrm{x}} \mathrm{S}$ nanoparticles acting as seeds for the formation of the $\mathrm{Cu}_{3} \mathrm{VS}_{4} \mathrm{NCs}$ by reacting with the $\mathrm{VO}(\text { acac })_{2}$ or $\mathrm{V}$-containing species. To assess the validity of this statement, presynthesized $\mathrm{Cu}_{\mathrm{x}} \mathrm{S}$ were directly reacted with the $\mathrm{VO}(\mathrm{acac})_{2}$ in the same reaction conditions and $\mathrm{Cu}_{3} \mathrm{VS}_{4}$ formed also in this case (Figure $\mathrm{S}_{4}$ ). The same $\mathrm{Cu}_{\mathrm{x}} \mathrm{S}$-seeded mechanism was observed for the heat-up synthesis, though accompanied by high polydispersity of the final samples, indicating that the decoupling of the nucleation and growth stages did not occur (Figure $\mathrm{S}_{5}$ ). Furthermore, $\mathrm{Cu}_{1.8} \mathrm{~S}$ impurities were found in one previous work on $\mathrm{Cu}_{3} \mathrm{VS}_{4}$ nanoparticles, which is also consistent with the formation of copper sulfide as an intermediate. ${ }^{25}$ In seed-mediated growth, two mechanisms are possible: dissolution/precipitation and solid state transformations. The latter are quite common across the literature with many examples reported on ternary metal phosphides nanoparticles by Brock et al. $^{41-44}$ Recently, we have also explained the formation of copper vanadate from $\mathrm{Cu} \mathrm{NC}$ seeds by demonstrating a solid state reaction between $\mathrm{Cu} \mathrm{NCs}$ and $\mathrm{VO}_{\mathrm{x} .}{ }^{38}$ Furthermore, presynthesized nanoparticles can be reacted to form ternary compounds, as shown by Dukovic et al for the synthesis of $\left(\mathrm{Ga}_{1-}\right.$ $\left.{ }_{\mathrm{x}} \mathrm{Zn}\right)\left(\mathrm{N}_{1-\mathrm{x}} \mathrm{O}_{\mathrm{x}}\right)$ nanoparticles obtained by nitriding a mixture of $\mathrm{ZnGa}_{2} \mathrm{O}_{4}$ and $\mathrm{ZnO}$ nanoseeds. ${ }^{45}$ To get further insights into the reaction between the $\mathrm{Cu}_{\mathrm{x}} \mathrm{S}$ seeds and the vanadium precursor, STEM-EDX analysis was performed on the reaction product obtained after 5 minutes from the DDT/OLAM injection and the results are reported in Figure 5 and Figure S6. The analysis evidences the presence of $\mathrm{Cu}_{\mathrm{x}} \mathrm{S}$ in the form of hexagonal nanoplateles and nanospheres, of $\mathrm{Cu}_{3} \mathrm{VS}_{4}$ nanocubes and of $\mathrm{V}$ containing nanoparticles (V-NPs). The latter are not detected by XRD, thus are probably mostly amorphous. At the moment, we do not have further information regarding the composition (Figure S6). This observation, together with the changes in $\mathrm{XRD}$ and TEM described above, suggests that a nanoscale solid state reaction is taking place between $\mathrm{Cu}_{\mathrm{x}} \mathrm{S}$ and the $\mathrm{V}$ containing NPs to form $\mathrm{Cu}_{3} \mathrm{VS}_{4}$. A sketch of the formation mechanism in depicted in Figure 6. In the case of a seed-mediated solid state reaction, templating effect are often observed, meaning that the size and shape of the seeds impacts the size and shape of the final product. ${ }^{3,41-44}$ In the present case, such correlation is not found, which might be due to the fast conversion kinetics. This conclusion is only speculative at the moment and will be investigated in the future with properly designed in-situ experiments combining X-Ray scattering and spectroscopies. ${ }^{38}$

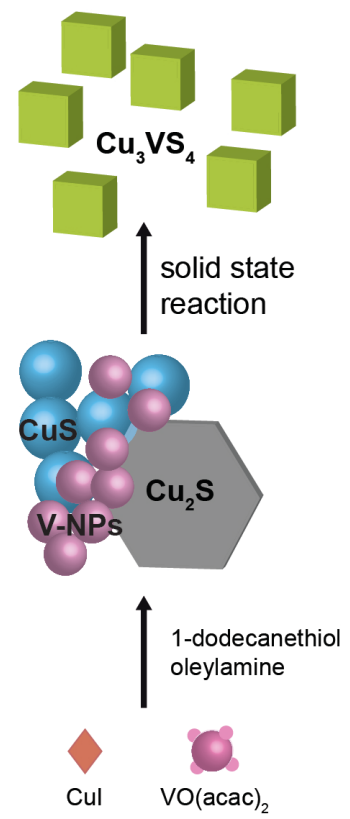


Figure 6. Sketch of the formation mechanism of the $\mathrm{Cu}_{3} \mathrm{VS}_{4}$ NCs.

The role of the ligands was carefully investigated having in mind their double role as modulators of the NCs surface energy and of the molecular precursor reactivity. Under the described reaction conditions, $\mathrm{CuI}, \mathrm{VO}(\text { acac })_{2}$ and DDT are the essential reagents for the reaction to proceed towards $\mathrm{Cu}_{3} \mathrm{VS}_{4}$. The addition of TOP contributes to modulate the $\mathrm{Cu}$ precursor reactivity by forming a $\mathrm{Cu}$-TOP complex and it strongly contributes to size control and monodispersity (Figure $\mathrm{S}_{7}$ ). On the other side, OLAM is key for shape control which other amines or carboxylic acids fail to provide (Figure S8). In agreement with these results, FTIR evidences that OLAM is the main ligand on the surface (Figure S9). The effect of using a lower or higher OLAM amount was also investigated and aggregated NCs or with a rectangular shape were obtained, respectively (Figure S10).

\section{Optical properties: experiments and theory}

As discussed in the introduction, $\mathrm{Cu}_{3} \mathrm{VS}_{4}$ is known to be an IB semiconductor but it has not been synthesized before in the form of NCs with tunable size. Thus, it becomes interesting to evaluate if any size-dependent behavior emerges in the optical properties. Figure $7 \mathrm{~A}$ shows the steady state UV-Vis spectra in solution for the three different sizes. They all exhibit a broad absorption onset in the near infrared (NIR) region which extends through the whole visible range $(780-380 \mathrm{~nm})$ where three peaks can be identified. These three peaks are all blueshifting with decreasing size, yet to a different extent. The high background in the spectra cannot be ascribed to scattering, considering that the solutions are very homogenous. A similar feature was also observed in $\mathrm{CuFeS}_{2} \mathrm{NCs}_{\text {and }}$ it was assigned to the electronic transitions occurring from the VB to the IB. ${ }^{20}$ The complexity of the electronic structure of IB-NCs motivated us to carry out DFT calculations to correlate the optical properties with the size and composition of the $\mathrm{Cu}_{3} \mathrm{VS}_{4}$ $\mathrm{NCs}$, considering that this aspect has not been carefully explored in any of the previous literature on IB-NCs. ${ }^{20,27,28,35}$ The results of these computations are reported in Figure 7 and in the Supporting Information (Figures $\mathrm{S}_{11}-14, \mathrm{~S}_{17}, \mathrm{~S} 18$ ). The presence of three peaks in the calculated absorption spectrum of bulk $\mathrm{Cu}_{3} \mathrm{VS}_{4}$ is in nice agreement with the experimental data (Figure ${ }_{7} \mathrm{~B}$ ). The electronic transitions mainly contributing to the three peaks have been visualized in Figures ${ }_{7} \mathrm{C}$ and S1o. All peaks correspond to transitions between states in the VB and the IB. The CB lies much higher in energy and transitions involving $\mathrm{CB}$ states would appear at much lower wavelengths in the UV. In order to investigate the peak shift with size, the DOS of two $\mathrm{Cu}_{3} \mathrm{VS}_{4} \mathrm{NCs}$ of $1.6 \mathrm{~nm}$ and $2.3 \mathrm{~nm}$ were calculated (Figure S12). These NCs are bounded by $\{001\}$ surfaces and the topmost layer is entirely $\mathrm{Cu}$, as predicted from surface-energy calculations (Figure $\mathrm{S}_{13}$ ) and consistently with the presence of a $\mathrm{Cu}$-rich surface. An opening of the band gap was found when decreasing the NC size (Figure S12). The functional form of the Brus equation was fitted to these theoretical results (Figure $\mathrm{S} 14$ ) to predict the band gap for the synthesized $\mathrm{Cu}_{3} \mathrm{VS}_{4} \mathrm{NCs}$. As a first approximation, the changes in the optical transitions between the valence and the intermediate bands were described by the rigid change in band gap as defined by the functional form of the Brus equation. Figure $7 \mathrm{D}$ reports both the experimental and theoretical peak shifts versus the NC size, where the $\Delta \lambda$ was calculated against a reference sample prepared by annealing the $\mathrm{Cu}_{3} \mathrm{VS}_{4} \mathrm{NCs}$ under nitrogen (Figures $\mathrm{S}_{15}$ and $\mathrm{S}_{16}$ ). While the theoretical trend is qualitatively followed by all the three peaks, only peak "I" matches the predicted $\Delta \lambda$ values while peak "II" \& "III" exhibit larger blue-shift than what predicted. Explaining the size-dependent optical behavior of IB semiconductors at the nanoscale is not trivial. In addition to classical quantum confinement described by the Brus equation, changes of the electronic structure from bulk to nano and possible effects from non-stoichiometric composition should be taken into account. The influence of the $\mathrm{Cu}$-rich composition on the optical transitions was excluded for the $\mathrm{Cu}_{3} \mathrm{VS}_{4} \mathrm{NC}$ s because the surface $\mathrm{Cu}$ atoms were found to contribute in a similar manner to those in the bulk to the PDOS of the NCs (Figure $\mathrm{S}_{17}$ ). While a Cu-rich surface is different than $3 \mathrm{ML} \mathrm{Cu}$ shell, the agreement between the calculated and experimental spectra in Figure $7 \mathrm{~A}, \mathrm{~B}$ suggests that the $\mathrm{Cu}$ shell does not have a drastic impact on the optical properties. A non-stoichiometric composition due to $\mathrm{Cu}$ interstitial would instead give rise to a red-shift of the peaks with decreasing size (Figure S18), which is opposite to what observed. Having ruled out the contribution of excess $\mathrm{Cu}$ atoms, whether at the interstitials or surface sites, to the optical properties of the $\mathrm{Cu}_{3} \mathrm{VS}_{4} \mathrm{NCs}$, the observed peak shifts must be due to the changes of the electronic structure going from bulk to nano (Figure S11). If the optical transitions are approximated with the ones known from the bulk, the calculated shifts from the 
A

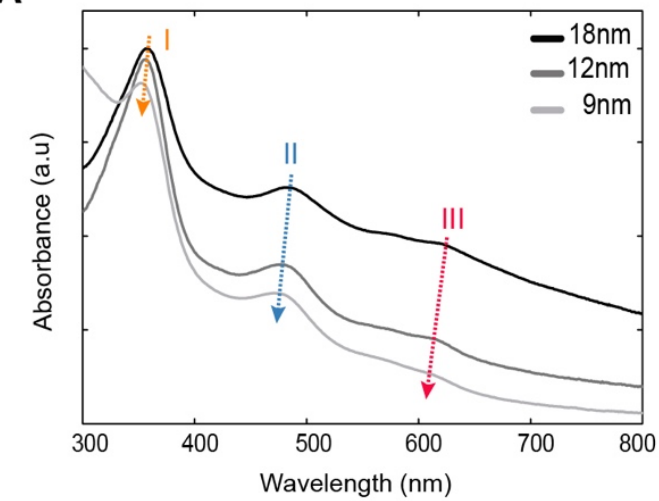

C

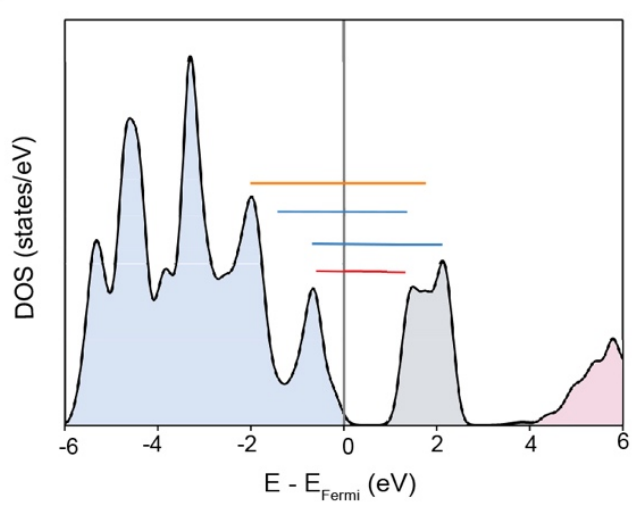

B

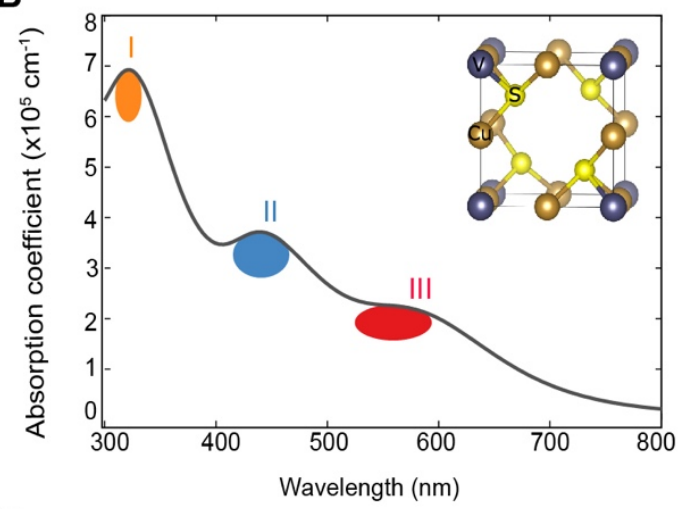

D

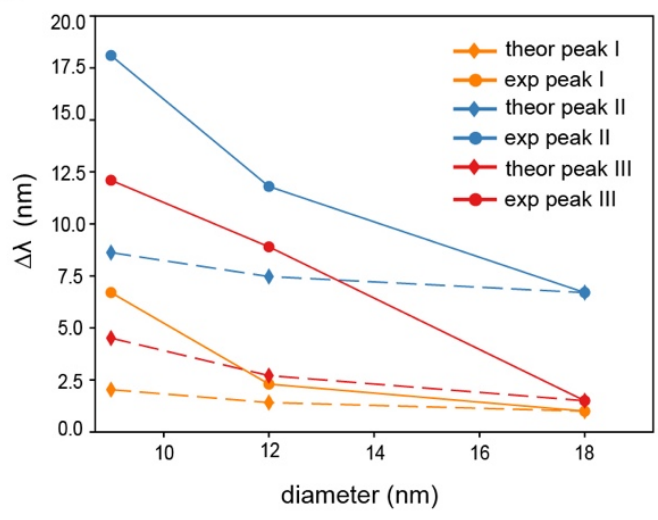

Figure 7. A) Steady-state UV-Vis absorption spectra of the $\mathrm{Cu}_{3} \mathrm{VS}_{4} \mathrm{NCs}$ with different sizes. The absorption peaks were off-set for clarity. B) Simulated absorption spectrum of bulk $\mathrm{Cu}_{3} \mathrm{VS}_{4}$. C) Density of states of bulk $\mathrm{Cu}_{3} \mathrm{VS}_{4}$ with the optical transitions, indicated by the horizontal lines between the states in the valence (blue) and intermediate (grey) bands. D) Shift of the absorption peaks as a function of size. The reference sample was prepared by annealing the $\mathrm{Cu}_{3} \mathrm{VS}_{4} \mathrm{NCs}$ in nitrogen at high temperature (Figure $\mathrm{S}_{15}$ )

model NCs have an opposite trend compared to the experimental data (Table S2). To reconcile this apparent inconsistency, it should be considered that the electronic structure of semiconductor NCs can be greatly influenced by the surface chemical environment (i.e. surface ligands type and concentration), something which is not included here at the moment. ${ }^{37,38}$ Deep-lying states, which give rise to transition "I", are expected to be less affected by chemical alterations compared to states at the band edges. This would explain why peak II and III do not match the calculated $\Delta \lambda$ values. The inclusion of ligand-induced effects in the band structure calculations will be helpful in the future to fully explain the optoelectronic properties of IB semiconductor NCs.

\section{Conclusions}

In this work, we have developed a hot injection method to synthesize colloidal $\mathrm{Cu}_{3} \mathrm{VS}_{4} \mathrm{NCs}$ with cubic shape and with tunable size ( $9 \mathrm{~nm}, 12 \mathrm{~nm}$ and $18 \mathrm{~nm}$ ). The corresponding steadystate UV-Vis spectra show three distinct absorption peaks which blue-shift with decreasing the NC size. Being the first detailed study on size-dependent optoelectronic properties in IB NCs, DFT calculations were utilized to explain the experimental data. Quantum confinement effects qualitatively account for the blue shifts observed in the optical spectra.
Changes of the electronic structures, including both size and chemical environment, must be considered to more closely match experiments and theory in the future. By introducing a novel class of IB NCs and investigating their size-dependent optical properties, this study contributes on one side to expand the chemistry of multinary semiconductor materials and on the other side to deepen the fundamental knowledge of how materials properties change at the nanoscale.

\section{ASSOCIATED CONTENT}

Additional experiments to validate the reaction mechanism (XRD, TEM, FTIR) and DFT calculations are reported in the Supporting Information.

This material is available free of charge via the Internet at http://pubs.acs.org

\section{AUTHOR INFORMATION}

\section{Corresponding Authors}

*E-mail: raffaella.buonsanti@epfl.ch 


\section{Notes}

The authors declare no conflict of interest.

\section{Acknowledgements}

This work was supported by the Swiss National Science Foundation (AP Energy Grant, project number PYAPP2_166897/1 and the SNF Professorship Grant PPooP2_157615). All calculations were performed on UBELIX (http://www.id.unibe.ch/hpc), the HPC cluster at the University of Bern. We thank Dr. Michal Strach for critical discussions and Dr Mounir Mensi for his help with XPS data acquisition.

\section{References}

(1) Norris, D. J.; Bawendi, M. G. Measurement and Assignment of the Size-Dependent Optical Spectrum in CdSe Quantum Dots. Phys. Rev. B 1996, 53, 16338-16346.

(2) Kovalenko, M. V; Manna, L.; Cabot, A.; Hens, Z.; Talapin, D. V; Kagan, C. R.; Klimov, X. V. I.; Rogach, A. L.; Reiss, P.; Milliron, D. J.; et al. Prospects of Nanoscience with Nanocrystals. ACS Nano 2015, 9, 1012-1057.

(3) Smith, A. M.; Nie, S. Semiconductor Nanocrystals: Structure, Properties, and Band Gap Engineering. Acc. Chem. Res. 2010, 43, 190200.

(4) Valerini D, Creti A., Lomascolo M., Manna L., Cingolani R., A. M. Temperature Dependence of the Photoluminescence Properties of Colloidal CdSe / ZnS Core / Shell Quantum Dots Embedded in a Polystyrene Matrix. Phys. Rev. B 2005, 71, 235409.

(5) Chem, J. M.; Park, J.; Kim, S. CuInS2/ZnS Core/Shell Quantum Dots by Cation Exchange and Their Blue-Shifted Photoluminescence. J. Mater. Chem. 2011, 21, 3745-3750.

(6) Coughlan, C.; Ibáñez, M.; Dobrozhan, O.; Singh, A.; Cabot, A.; Ryan, K. M. Compound Copper Chalcogenide Nanocrystals. Chem. Rev. 2017, 117, 5865-6109.

(7) Knowles, K. E.; Hartstein, K. H.; Kilburn, T. B.; Marchioro, A.; Nelson, H. D.; Whitham, P. J.; Gamelin, D. R. Luminescent Colloidal Semiconductor Nanocrystals Containing Copper: Synthesis, Photophysics, and Applications. Chem. Rev. 2016, 116, 10820-10851.
(9) Soc. Rev. 2013, 42, 2986-3017.

Energy Conversion and Storage Devices. Chem.

Kolny-Olesiak, J.; Weller, H. Synthesis and Application of Colloidal CuInS2 Semiconductor Nanocrystals. ACS Appl. Mater. Interfaces 2013, No. 23, 12221-12237.

(10) Leach, A. D. P.; Mast, L. G.; Hernández-Pagán, E. A.; Macdonald, J. E. Phase Dependent Visible to Near-Infrared Photoluminescence of CuInS2 Nanocrystals. J. Mater. Chem. C 2015, 3, 32583265.

(11) Cuins, M. P.; Zhong, H.; Lo, Ḱ. S. S.; Mirkovic, T.; Li, Y.; Ding, Y.; Li, Y.; Scholes, G. D. Noninjection Gram-Scale Synthesis of Monodisperse Pyramidal CuInS2 Nanocrystals and Their SizeDependent Properties. ACS Nano 2010, 4, 52535262.

(12) Protesescu, L.; Yakunin, S.; Bodnarchuk, M. I.; Krieg, F.; Caputo, R.; Hendon, C. H.; Yang, R. X.; Walsh, A.; Kovalenko, M. V. Nanocrystals of Cesium Lead Halide Perovskites ( $\mathrm{CsPbX}_{3}, \mathrm{X}=$ $\mathrm{Cl}, \mathrm{Br}$, and I): Novel Optoelectronic Materials Showing Bright Emission with Wide Color Gamut. Nano Lett. 2015, 15, 3692.

(13) A. Swarnkar, A. R. Marshall, E. M. Sanehira, B. D. Chernomordik, D. T. Moore, J. A. Christians, T. Chakrabarti, J. M. L. Quantum Dot-induced Phase Stabilization of $\mathrm{A}_{-} \mathrm{CsPb}_{3}$ Perovskite for High-Efficiency Photovoltaics. Science (80-. ). 2016, 354, 92-95.

(14) Wheeler, L. M.; Sanehira, E. M.; Marshall, A. R.; Schulz, P.; Suri, M.; Anderson, N. C.; Christians, J. A.; Nordlund, D.; Sokaras, D.; Kroll, T.; et al. Targeted Ligand Exchange Chemistry on Cesium Lead Halide Perovskite Quantum Dots for High-Efficiency Photovoltaics. J. Am. Chem. Soc. 2018, 140, 10504-10513.

(15) Vörös, M.; Galli, G.; Zimanyi, G. T. Colloidal Nanoparticles for Intermediate Band Solar Cells. ACS Nano 2015, 9, 6882-6890.

(16) Luque, A.; Martí, A. Increasing the Efficiency of Ideal Solar Cells by Photon Induced Transitions at Intermediate Levels. Phys. Rev. Lett. 1997, 78, 5014-5017.

(17) Hanna, M. C.; Nozik, A. J. Solar Conversion Efficiency of Photovoltaic and Photoelectrolysis Cells with Carrier Multiplication Absorbers. J. Appl. Phys. 2006, 100, 074510.
Gao, M. R.; Xu, Y. F.; Jiang, J.; Yu, S. H. Synthesis, Modification, and Applications in 
(18) Luque, A.; Martí, A.; Stanley, C. Understanding Intermediate-Band Solar Cells. Nat. Photonics 2012, 6, 146-152.

(19) Okada, Y.; Ekins-Daukes, N. J.; Kita, T.; Tamaki, R.; Yoshida, M.; Pusch, A.; Hess, O.; Phillips, C. C.; Farrell, D. J.; Yoshida, K.; et al. Intermediate Band Solar Cells: Recent Progress and Future Directions. Appl. Phys. Rev. 2015, 2 (2), 021302.

(20) Ghosh, S.; Avellini, T.; Petrelli, A.; Kriegel, I.; Gaspari, R.; Almeida, G.; Bertoni, G.; Cavalli, A.; Scotognella, F.; Pellegrino, T.; et al. Colloidal CuFeS2 Nanocrystals: Intermediate Fe d-Band Leads to High Photothermal Conversion Efficiency. Chem. Mater. 2016, 28, 4848-4858.

(21) Lucena, R.; Aguilera, I.; Palacios, P.; Wahnón, P.; Conesa, J. C. Synthesis and Spectral Properties of Nanocrystalline V-Substituted $\mathrm{In}_{2} \mathrm{~S}_{3}$, a Novel Material for More Efficient Use of Solar Radiation. Chem. Mater. 2008, 20, 5125-5127.

(22) Chen, P.; Qin, M.; Chen, H.; Yang, C.; Wang, Y.; Huang, $\mathrm{F}$. $\mathrm{Cr}$ Incorporation in $\mathrm{CuGaS}_{2}$ Chalcopyrite: A New Intermediate-Band Photovoltaic Material with Wide-Spectrum Solar Absorption. Phys. Status Solidi Appl. Mater. Sci. 2013, 210, 1098-1102.

(23) Kehoe, A. B.; Scanlon, D. O.; Watson, G. W. Modelling Potential Photovoltaic Absorbers $\mathrm{Cu}_{3} \mathrm{MCh}_{4}(\mathrm{M}=\mathrm{V}, \mathrm{Nb}, \mathrm{Ta}$; $\mathrm{Ch}=\mathrm{S}$, Se, Te) Using Density Functional Theory. J. Phys. Condens. Matter 2016, 28, 175801.

(24) Kehoe, A. B.; Scanlon, D. O.; Watson, G. W. The Electronic Structure of Sulvanite Structured Semiconductors $\mathrm{Cu}_{3} \mathrm{MCh}_{4}(\mathrm{M}=\mathrm{V}, \mathrm{Nb}, \mathrm{Ta} ; \mathrm{Ch}=$ $\mathrm{S}$, Se, Te): Prospects for Optoelectronic Applications. J. Mater. Chem. C 2015, 3, 1223612244 .

(25) Chen, C. C.; Stone, K. H.; Lai, C. Y.; Dobson, K. D.; Radu, D. Sulvanite (Cu3VS4) Nanocrystals for Printable Thin Film Photovoltaics. Mater. Lett. 2018, 211, 179-182.

(26) Lv, X. S.; Deng, Z. H.; Miao, F. X.; Gu, G. X.; Sun, Y. L.; Zhang, Q. L.; Wan, S. M. Fundamental Optical and Electrical Properties of Nano$\mathrm{Cu}_{3} \mathrm{VS}_{4}$ Thin Film. Opt. Mater. (Amst). 2012, 34, 1451-1454.

(27) Gaspari, R.; Della Valle, G.; Ghosh, S.; Kriegel, I.; Scotognella, F.; Cavalli, A.; Manna, L. QuasiStatic Resonances in the Visible Spectrum from All-Dielectric Intermediate Band
Semiconductor Nanocrystals. Nano Lett. 2017, 17, 7691-7695.

(28) Sugathan, A.; Bhattacharyya, B.; Kishore, V. V. R.; Kumar, A.; Rajasekar, G. P.; Sarma, D. D.; Pandey, A. Why Does CuFeS2 Resemble Gold?J. Phys. Chem. Lett. 2018, 9, 696-701.

(29) Xia, Z.; Fang, H.; Zhang, X.; Molokeev, M. S.; Gautier, R.; Yan, Q.; Wei, S. H.; Poeppelmeier, K. R. CsCu5Se3: A Copper-Rich Ternary Chalcogenide Semiconductor with Nearly Direct Band Gap for Photovoltaic Application. Chem. Mater. 2018, 30, 1121-1126.

(30) Kresse, G.; Furthmuller, J. Efficient Iterative Schemes for Ab Initio Total Energy Calculations Using a Plane-Wave Basis Set. Phys. Rev. B 1996, 54, 11169-11186.

(31) Kresse, G.; Joubert, D. From Ultrasoft Pseudopotentials to the Projector Augmented Wave Method. Phys. Rev. B 1999, 59, 1758-1775.

(32) Perdew, J. P.; Ruzsinszky, A.; Csonka, G. I.; Vydrov, O. A.; Scuseria, G. E.; Constantin, L. A.; Zhou, X.; Burke, K. Restoring the DensityGradient Expansion for Exchange in Solids and Surfaces. Phys. Rev. Lett. 2008, 100, 136406.

(33) Petritis D., Martinez G., Levy-Clement C., G. O. Investigation of the Vibronic Properties of $\mathrm{Cu}_{3} \mathrm{VS}_{4}, \mathrm{Cu}_{3} \mathrm{NbS}_{4}$, and $\mathrm{Cu}_{3} \mathrm{TaS}_{4}$ Compounds. 1981, pp 6773-6786.

(34) Mujica, C.; Carvajal, G.; Llanos, J. Redetermination of the Crystal Structure of Copper (I) Tetrathiovanadate (Sulvanite), $\mathrm{Cu}_{3} \mathrm{VS}_{4}$. Zeitschrift für Krist. - New Cryst. Struct. 1998, 213, 12.

（35） Scanlon, D. O.; Morgan, B. J.; Watson, G. W. Modeling the Polaronic Nature of $p$-Type Defects in $\mathrm{Cu}_{2} \mathrm{O}$ : The Failure of GGA and GGA+U. J. Chem. Phys. 2oo9, 131, 124703.

(36) Arribart, H.; Sapoval, B.; Gorochov, O.; LeNagard, N. Fast Ion Transport at Room Temperature in the Mixed Conductor $\mathrm{Cu}_{3} \mathrm{VS}_{4}$. Solid State Commun. 1978, 26, 435-439.

（37） Dai, Q.; Wang, Y.; Li, X.; Zhang,Y,; Pellegrino, D.J.; Zhao,M.; Zou. B.; Seo,J.T.; Wang,Y.; Yu, W. W. Size-Dependent Composition and Molar Extinction Coefficient of PbSe Semiconductor Nanocrystals. ACS Nano 2009, 3, 1518-1524.

(38) Gadiyar, C.; Strach, M.; Schouwink, P.; Loiudice, A.; Buonsanti, R. Chemical Transformations at 
the Nanoscale: Nanocrystal-Seeded Synthesis of $\beta-\mathrm{Cu}_{2} \mathrm{~V}_{2} \mathrm{O}_{7}$ with Enhanced Photoconversion Efficiencies. Chem. Sci. 2018, 9, 5658-5665.

（39） Nenashev, R. N.; Mordvinova, N. E.; Zlomanov, V. P.; Kuznetsov, V. L. Thermal Decomposition of Vanadyl Acetylacetonate. Inorg. Mater. 2015, 51, 891-896.

(40) Su, D. W.; Dou, S. X.; Wang, G. X. Hierarchical Orthorhombic $\mathrm{V}_{2} \mathrm{O}_{5}$ Hollow Nanospheres as High Performance Cathode Materials for Sodium-Ion Batteries. J. Mater. Chem. A 2014, 2 (29), 11185-11194.

(41) Li, D.; Arachchige, M. P.; Kulikowski, B.; Lawes, G.; Seda, T.; Brock, S. L. Control of Composition and Size in Discrete CoxFe2-XP Nanoparticles: Consequences for Magnetic Properties. Chem. Mater. 2016, 28, 3920-3927.

(42) Liyanage, D. R.; Danforth, S. J.; Liu, Y.; Bussell, M. E.; Brock, S. L. Simultaneous Control of Composition, Size, and Morphology in Discrete Ni2-XCoxP Nanoparticles. Chem. Mater. 2015, $27,4349-4357$.

(43) Muthuswamy, E.; Brock, S. L. Solid-State Phase Transformations in Solution: Templated Conversion of Nanoscale Nickel Phosphides.
Chem. Commun. 2011, 47, 12334-12336.

(44) Hitihami-Mudiyanselage, A.; Arachchige, M. P.; Seda, T.; Lawes, G.; Brock, S. L. Synthesis and Characterization of Discrete FexNi2- XP Nanocrystals $(\mathrm{o}<\mathrm{X}<2)$ : Compositional Effects on Magnetic Properties. Chem. Mater. 2015, 27, 6592-66oo.

(45) Tongying, P.; Lu, Y. G.; Hall, L. M. G.; Lee, K.; Sulima, M.; Ciston, J.; Dukovic, G. Control of Elemental Distribution in the Nanoscale SolidState Reaction That Produces (Ga1-XZnx)(N1XOx) Nanocrystals. ACS Nano 2017, 11, 84018412. 